\title{
Chaperones as Buffering Agents?
}

\section{Thomas Mitchell-Olds and Charles A. Knight}

D oes evolution draw on existing genetic variation in animals and plants or must it wait around for new mutations to arise? Sixty years ago, Waddington argued that cryptic genetic variation is present for many traits, but that expression of these variants under normal environmental conditions is prevented by a process of "genetic buffering" (1). As Waddington demonstrated, stressful environmental conditions compromise the genetic buffering system, leading to the breakdown of normal development and enabling the expression of cryptic genetic variation as visible phenotypic changes. Recently, heat shock proteins (HSPs), a type of molecular chaperone, have been implicated in the genetic buffering of the fruit fly Drosophila. Now, Queitsch et al. (2) report in a recent issue of Nature that the chaperone HSP90 provides genetic buffering in Arabidopsis and may contribute to the evolutionary adaptation of this plant.

HSPs are induced by high-temperature stress in organisms as diverse as bacteria, fungi, plants, and animals. These molecular chaperones prevent irreversible aggregation of denatured proteins after heat or other protein-denaturing stresses. They also bind to a range of client proteins that are crucial for regulating growth and development. The evolutionary conservation of the heat shock response, and 
the fact that expression of HSPs correlates with high-temperature stress suggests that accumulation of HSPs increases thermotolerance. This prediction is borne out by numerous studies in animal and plant cells. For example, Arabidopsis plants that overexpress HSP101 exhibit increased resistance to heat stress (3). Furthermore, after heat stress a low molecular weight HSP in plant chloroplasts stabilizes photosystem II proteins that produce oxygen, and also maintains electron transport in photosystem II (4). There are strong correlations between the heat shock response and survival (5), specific leaf area (6), and other ecologically important traits. However, without genetic or biochemical experiments, it is difficult to calculate the extent to which HSPs contribute to genetic buffering in plants.

In Drosophila, natural genetic variation and mutations induced by genetic engineering reveal that HSP70 is important for inducing thermotolerance. Transgenic flies that overexpress HSP70 are better able to resist heat stress (7). Natural genetic variants (polymorphisms) in wild fly populations can be selected for in the laboratory. For example,

Bettencourt et al. (8) selected genetic variants of the HSP70 promoter in Drosophila that correlated with altered regulation of HSP70 gene expression. They found that different variants rose to a high frequency in five laboratory fruit fly populations that had been grown at different temperatures for 20 years. Intriguingly, these variants showed similar patterns of allele frequencies along a latitudinal transect of Australia with a wide variation in climate. Both studies suggest that environmental temperature stresses result in selection of particular HSP70 variants in both wild and laboratory fruit fly populations.

In both animal and plant cells, the HSP90 chaperone has a limited number of crucial client proteins that it keeps in a state of activation, including steroid hormone receptors, signaling kinases, and a variety of transcription factors (9). In the fruit fly, pharmacological blockade of HSP90 activity causes selective inactivation or degradation of these client proteins, resulting in developmental abnormalities and numerous morphological changes in many structures. Likewise, HSP90 inhibitors display antitumor activity, altering the expression of cell cycle genes (10). Given their direct interac- tions with signal transduction proteins and other regulatory proteins, HSPs are predicted to influence physiological and morphological variations, and hence are good candidate genetic buffering molecules.

In a previous study in fruit flies, Rutherford and Lindquist (11) revealed that HSP90 buffers against expression of genetic variation by stabilizing the protein constituents of signal transduction pathways. When HSP90 activity was impaired either by mutation or by pharmacological blockade, a considerable number of phenotypic variants appeared. The authors selected several phenotypic variants in the HSPcompromised flies over a few generations. They found that the new morphological traits were expressed in flies even when HSP90 activity was restored to wild-type levels (an example of genetic assimilation). Furthermore, under high-temperature stress conditions, these same morphological variants appeared in wild-type fruit fly populations, presumably because HSP90 had been recruited to the heat shock response and was no longer available to buffer the regulatory pathways influencing these phenotypes. Rutherford and Lindquist argued that the buffering capacity of HSP90 could contribute to evolution.

In their new work, Lindquist and co-workers (2) chose a completely different organism, Arabidopsis, in which to seek a parallel example of HSP90 buffering capacity with similar evolutionary implications. In contrast to animals, plant development is highly plastic and intimately connected with environmental cues. For several strains of Arabidopsis, pharmacological or heat-induced reductions in HSP90 activity produced a variety of morphological variants, such as those with altered color and presence of true leaves, shape and expansion of cotyledons, root morphology, and orientation of seedlings. These developmental abnormalities were strain-specific, suggesting that HSP90 does indeed buffer hidden genetic variation. Lindquist and colleagues propose that HSP90 regulates release of hidden genetic variation, generating morphological changes that may contribute to adaptive evolution. However, the evolutionary potential of newly revealed genetic variation may differ between Drosophila and Arabidopsis. HSP90 inactivation results in grossly abnormal morphologies and reduced survival in Drosophila, hence these cryptic genetic variants may not be evo- lutionarily advantageous. In contrast, HSP90 blockade in Arabidopsis induced numerous morphological variants with good survival frequencies in the laboratory.

Although genetic and pharmacological manipulation of HSP90 in Drosophila and Arabidopsis in the laboratory unveils cryptic genetic variation, the crucial question is whether extant hidden genetic variation actually contributes to evolutionary change in the field. A recent study by Lauter and Doebley in maize (12) suggests that it may. These authors analyzed three morphological traits that distinguish maize from its wild ancestor, teosinte, but that are uniform within ancestral teosinte populations (see the figure). Quantitative trait locus (QTL) mapping of variations in a testcross teosinte population identified 22 QTLs controlling morphological traits that are normally invariant in teosinte populations. Thus, the evolution of modern maize may have drawn upon cryptic genetic variation that already existed in teosinte.

HSPs are excellent candidates for the genetic buffering system that Waddington predicted in the 1940s. The Lindquist laboratory $(2,11)$ has certainly demonstrated that temperature stress or HSP inhibition reveals potentially adaptive variation. But do HSPs actually buffer adaptive genetic variation in the field, and does release of this variation drive adaptation? To address these questions, evolutionary biologists will need to show that cryptic variation can be adaptive (as suggested by the work of Lauter and Doebley), and must also demonstrate that HSP90 is responsible for keeping this cryptic variation hidden. This will require experiments demonstrating that adaptive evolution differs between HSP-wild-type and HSP-compromised plant or fruit fly populations.

It is clear that HSPs are important for regulating gene expression and for inducing thermotolerance. But we have much more to do if we want to obtain convincing evidence that breakdown of HSP-mediated buffering is an important contributor to adaptive evolution in animals or plants.

\section{References}

1. C. H. Waddington, Nature 150, 563 (1942)

2. C. Queitsch, T. A. Sangster, S. Lindquist, Nature 417, 618 (2002); advanced online publication, 12 May 2002 (10.1038/nature749)

3. C. Queitsch, S. Hong, E. Vierling, S. Lindquist, Plant Cell 12, 479 (2000).

4. S. A. Heckathorn, C. A. Downs, T. D. Sharky, J. S. Coleman, Plant Physiol. 116, 439 (1998).

5. S. K. Park, R. Chang, R. Shivaji, D. S. Luthe, Plant Physiol. 115, 229 (1997).

6. C. A. Knight, D. D. Ackerly, Am. J. Bot. 88, 411 (2001)

7. S. P. Roberts, M. E. Feder, Funct. Ecol. 14, 353 (2000).

8. B. R. Bettencourt, I. Y. Kim, A. A. Hoffmann, M. E. Feder, Evolution, in press.

9. J. C. Young, I. Moarefi, F. U. Hartl, J. Cell Biol. 154, 267 (2001).

10. L. Neckers, Trends Mol. Med. 8 (suppl.), 56 (2002)

11. S. L. Rutherford, S. Lindquist, Nature 396, 336 (1998).

12. N. Lauter, J. Doebley, Genetics 160, 333 (2002). 